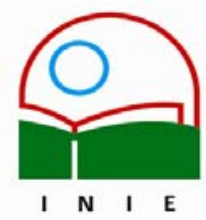

Actualidades Investigativas en Educación

Revista Electrónica publicada por el

Instituto de Investigación en Educación

Universidad de Costa Rica

ISSN 1409-4703

http://revista.inie.ucr.ac.cr

COSTA RICA

\title{
COMUNICACIÓN Y LENGUAJE EN PERSONAS QUE SE UBICAN DENTRO DEL ESPECTRO AUTISTA
}

COMMUNICATION AND LANGUAGE IN PEOPLE WHO ARE LOCATED WITHIN

PHANTOM AUTISTA

\author{
Volumen 7, Número 2 \\ Mayo-Agosto 2007
}

pp. 1-16

Este número se publicó el 30 de agosto 2007

Ronald Soto Calderón

La revista está indexada en los directorios:

LATINDEX,$\underline{\text { REDALYC}}$ IRESIE, $\underline{\text { CLASE}}$, DIALNET, DOAJ, E-REVIST@S,

La revista está incluida en los sitios:

REDIE, RINACE, OEI, MAESTROTECA, HUASCARAN 


\title{
COMUNICACIÓN Y LENGUAJE EN PERSONAS QUE SE UBICAN DENTRO DEL ESPECTRO AUTISTA \\ COMMUNICATION AND LANGUAGE IN PEOPLE WHO ARE LOCATED WITHIN \\ PHANTOM AUTISTA
}

\author{
Ronald Soto Calderón ${ }^{1}$
}

\begin{abstract}
Resumen: Una de las mayores dificultades de las personas que se ubican dentro del espectro autista, está relacionado con los elementos semántico pragmáticos de la comunicación, no obstante, quienes tienen relación con ellas en un afán de satisfacer sus necesidades procuran darle "entender lo que ellas dicen" sin tener en cuenta que muchas veces son meras interpretaciones propias, situación que afecta el desarrollo y utilización de un método o una estrategia de enseñanza del lenguaje y la comunicación a estas personas. Con este artículo se pretende realizar un corto recorrido por las características del lenguaje en personas con autismo, y algunos métodos que podrían favorecer el desarrollo de destrezas comunicativas en ellas.
\end{abstract}

Palabras claves: COMUNICACIÓN/ LENGUAJE/ PERSONAS CON AUTISMO/ MÉTODOS DE ENSEÑANZA DEL LENGUAJE Y COMUNICACIÓN/

\begin{abstract}
One of the biggest difficulties that lie in to the autistic spectre, is related to the semantic pragmatic elements of communication, nevertheless, those who are related to those fundamentals of communication, in the need to satisfy their needs procure giving "understanding to what they say" without realizing that most times those are nothing but insightful interpretations, which affect the development and method employment or a language teaching strategy and communication of such people. This article is intended to take a short look into the language characteristics from people with autism and some methods that might favour their communication skills development.
\end{abstract}

Key words: COMMUNICATION/ LANGUAGE/ PERSONS WITH AUTISM/ TEACHING METHODS FOR COMMUNICATION AND LANGUAGE/

\section{1.- A manera de introducción}

Cuando se habla de comunicación y lenguaje en personas con Autismo, nos embarcamos en una experiencia que involucra una serie de preguntas relacionadas con el desarrollo de estas personas, y como el trastorno generalizado del desarrollo afecta todo su proceso de desarrollo integral.

${ }^{1}$ Doctor en Educación de la Universidad de La Salle, Costa Rica. Magíster
en Educación Especial -ICSE- Universidad Pontificia de Comillas, Madrid,
España; Magíster en Evaluación Educativa de la Universidad de Costa
Rica; Subdirector y profesor de la Escuela de Orientación y Educación
Especial de la Universidad de Costa Rica; Profesor de la División de
Educación Básica del Centro de Investigación y Docencia en Educación de
la Universidad Nacional. Profesor de la Maestría en Estudios
Interdisciplinarios sobre Discapacidad; Investigador del Instituto de
Investigación para el Mejoramiento de la Educación Costarricense (IIMEC).

Correo electrónico: ronaldsc@cariari.ucr.ac.cr

Artículo recibido: 21 de febrero, 2007

Aprobado: 28 de agosto, 2007 
Los problemas de comunicación relacionados con el autismo varían según el desarrollo intelectual y social de la persona. Algunos son incapaces de hablar, mientras que otros tienen vocabularios ricos y pueden conversar acerca de temas de interés en gran profundidad. A pesar de esta diferencia, la mayoría de las personas con autismo tiene poco o ningún problema de pronunciación, otras de ellas tienen problemas para usar el idioma de manera eficaz, y muchos también tienen dificultades con el significado de la palabra y de la oración, la entonación y el ritmo.

Las personas con autismo que pueden hablar, a menudo dicen cosas que no tienen ningún contenido o información. Por ejemplo, una persona con autismo puede contar reiteradamente una serie de números, por ejemplo, de uno a cinco, estando en una situación donde esa actividad no tiene sentido. Otros hacen uso de la ecolalia, o sea la repetición de algo que se ha escuchado con anterioridad. En este sentido se puede mencionar,

a. una forma de ecolalia denominada inmediata, la cual ocurre cuando la persona repite la pregunta que le han hecho, por ejemplo, "¿cómo se llama usted?" y en lugar de contestar con el nombre, repite la pregunta que le han planteado.

b. otra forma de ecolalia se denomina retrasada, una personas puede preguntar, y esta consiste en que hacen uso de frases o de oraciones, o palabras, que han escuchado anteriormente, y en situaciones muchas veces ajenas a las que están viviendo en el momento, y las repiten en una situación completamente ajena, por ejemplo, de visita en casa de la abuela dos meses antes escucharon la palabra "gallina", y estando en clase con el resto de los compañeros hablando sobre el estado del tiempo, comienzan a decir "gallina" reiteradamente.

c. Muchas de las personas con autismo, hacen uso de frases que ya han sido almacenadas en su memoria para comenzar una conversación, por ejemplo, "me llamo Luis", no importa si están hablando con personas que conocen, frecuentan o familiares.

d. Otras personas con autismo, acostumbran repetir guiones que han aprendido o escuchado, en algunos momentos, por ejemplo, en los anuncios de la radio o la televisión.

e. Por otro lado se da el caso de que algunas personas con autismo con un grado mayor de inteligencia, pueden hablar exhaustivamente sobre temas en los que están muy interesados, por ejemplo, de un viaje, de cámaras de video, de animales 
salvajes, de carros, de juegos, pero se les dificulta participar en una conversación sostenida con otra persona acerca de la temática de interés

Es importante señalar que algunas personas con autismo no tienen contacto visual con sus interlocutores, y su atención es deficiente. Además la mayor parte de las veces se les dificulta el uso de gestos como una forma primaria de comunicación, por ejemplo, lenguaje de señas, o como un aspecto complementario al hecho comunicativo verbal señalando o mencionando un objeto al cual quieren tener acceso (Frith, 1989; Soto, 1994; Gortazar, 1993).

Algunas personas con autismo tienden a hablar en un tono de voz muy alto o en forma monótona, lo cual muchas veces se asemeja a un autómata o como un habla sintetizada Frith, 1989, Marchena, 1998).

Como producto de los aspectos señalados en los párrafos anteriores, se puede decir que las personas con autismo comúnmente no son receptivas a los actos comunicativos de los demás, y esto se dificulta muchas veces al no poder responder con sus propios nombres, esta situación puede provocar valoraciones inapropiadas de las otras personas, aun sus padres, quienes consideran que son sordos, o tienen dificultades auditivas (Tamarit, 1993).

Una de las mayores dificultades en el lenguaje de las personas con autismo es el uso correcto de los pronombres, lo cual también representa un grave problema en la comunicación para las personas con autismo, por ejemplo muchas veces expresa "quiere agua" cuando lo correcto sería "yo quiero agua" (Peeters, 1997; Soto, 1994).

Es importante señalar que muchas personas con autismo, desarrollan el habla y lenguaje, pero sólo hasta cierto nivel, muchas veces no alcanza un nivel adecuado para la edad. No obstante, ese desarrollo generalmente es desigual. Así por ejemplo, se puede acelerar el desarrollo de vocabulario en las aquellas áreas de interés puede verse acelerado, mientras que en otras áreas no se evidencia esa situación, esto se percibe principalmente en situaciones en las que demuestran buena memoria para recordar la información que acaban de escuchar o ver, pero no saben como hacer uso de ella. $Y$ algunos pueden leer mucho antes de los cinco años de edad, pero no demuestran comprensión de lo que leen (Schaeffer, 1986, Sotillo y Rivière, 1997). 
Como se puede deducir de las ideas anteriores, no es únicamente importante hablar de comunicación y lenguaje como una esfera de trabajo con las personas con autismo, sino que más bien es clave poner énfasis en la necesidad de que aquellos que trabajan con ellas, comprendan la necesidad de las interacciones efectivas y que es mediante estas y la comunicación que las personas con autismo pueden aprender, a interactuar en los diferentes escenarios en los cuales participan, tanto dentro de la escuela como en los diferentes entornos.

A menudo preocupa que las personas con autismo no puedan comunicarse, pero se deja de lado, el hecho de que el entorno en el cual se desarrollan esas personas debe también brindar respuestas a sus intenciones comunicativas, no es solamente darle sentido a lo que se "supone" quieren decir ellas; sino más bien es importante que esa "intención comunicativa" se vea reforzada por un accionar adecuado y con sentido por parte del adulto. No es dejarse llevar por la persona, es responder y darle sentido a lo que se hace (Schaeffer, 1986, Sotillo y Rivière, 1997, Soto, 1994).

Lo anterior, se propone teniendo en cuenta que las personas siempre prestan especial atención ante su cuidador, y que en un inicio dependen mucho de aquellos que le cuidan, con lo cual hay que tener cuidado, y evitar la dependencia permanente y la poca independencia que podrían desarrollar las personas con autismo (Gortazar, 1993).

De alguna manera, la iniciativa por comunicarse e interaccionar, aun cuando sea difícil de comprender siempre es propuesta desde el inicio por la persona con autismo, y eso es lo que debemos aprender a comprender, más que enseñarle a comunicarse a estas personas, muchas veces se debe aprender a escucharlas, a entender sus silencios, sus no, sus así estoy bien, sus expresiones, entre otros aspectos.

Como se puede ver, la comunicación y el lenguaje debe abordarse como algo natural, que poco a poco con la interacción se va convirtiendo en una interacción más consciente, favoreciendo de esta manera que estos dos procesos se conviertan en aspectos básicos para el desarrollo y el aprendizaje de la persona con autismo (Gortazar, 1993). 
La comunicación por lo tanto, debe verse como un proceso en el cual las personas comparten, y mediante ella se logra la interacción y la mutualidad, aspecto importante en la convivencia con los demás (Soto, 2002).

La comunicación en el caso de las personas con autismo por lo tanto, debe percibirse como el proceso que evita que las intenciones comunicativas y los hechos comunicativos pierdan todo efecto sobre las demás personas, y que estas aprendan a retroalimentar a las personas con autismo, para así favorecer sus interacciones con los demás.

Por las razones antes mencionadas, es que se deben desarrollar programas de comunicación con las personas con autismo, que le permitan una adecuada interacción con los demás, y que respondan a sus necesidades, intereses y particularidades, y que además respeten sus niveles de respuesta.

\section{2.- Comunicación y lenguaje ¿por qué trabajarlos en personas con autismo?}

Se debe recordar que por su condición discapacitante, las personas con autismo han tenido serias dificultades en su forma de interactuar y manipular el mundo físico, social y abstracto de una forma que sería vista como adecuada, y por esta razón muchas son las dificultades que encuentran en sus procesos de convivencia con los demás. Por otro lado, es difícil para estas personas tener claro conceptos como tiempo, espacio, cantidad, calidad y causalidad, lo cual siempre redundará en serias dificultades para el desarrollo de la comunicación.

Estas dificultades en la comprensión del mundo dificulta a las personas con autismo la toma de decisiones, se le dificultará poder tener acción o influir sobre su situación personal, sobre su vida, en fin llevar a cabo una vida independiente, se hace necesario aclarar aquí que el aprendizaje de un sistema de comunicación no evitará siempre la ocurrencia de estos procesos, pero si mejoraran la condición de vida de quienes portan este síndrome.

A menudo las dificultades que se encuentran cuando se quiere trabajar la comunicación en personas con autismo hacen referencia a

1. La persona por lo general no aprende las técnicas.

2. A menudo al no ver reforzadas sus intenciones comunicativas, prefiere alejarse del proceso, lo cual muchas veces redunda en las dificultades que encuentran para 
hacer uso de ellas, y el no sentirse motivado por interactuar con otras personas, y entonces no aprenderá.

3. Generalmente, la persona aprende a discriminar que en algunas ocasiones hará uso de sus intenciones comunicativas y en otras no, lo cual muchas veces afecta su espontaneidad y disposición comunicativa, hecho que no solo afecta a la persona, sino también a aquellos que conviven con ella.

Por estas razones, las personas que interactúan con los portadores del Síndrome Autista, generalmente se confunden por el tipo de interacciones que ellos intentan tener, ya que los desórdenes de tipo perceptual o motor de estas personas no tienen ningún sentido para ellas.

A menudo las manifestaciones expresivas que las personas con Autismo utilizan no corresponden con aquellas que quienes interactúan con estas personas están acostumbrados a ver en sus interlocutores.

De esta manera, el trabajo en comunicación con las personas con autismo debe responder a las formas de comunicación más natural que para ellos representan, teniendo siempre en cuenta que la forma de comunicación elegida le debe permitir a la persona con autismo experimentar, entender y practicar la comunicación, y que además permitan ver la funcionalidad de la intención comunicativa, es interesante anotar aquí que muchas veces la elección y trabajo mediante un método o modelo de comunicación ha permitido a personas con autismo (y otras condiciones discapacitantes) iniciar un proceso de comunicación oral, situación que puede asociarse a que ellas de alguna manera logran una satisfacción de necesidades como respuesta a una intención comunicativa (Gortazar, 1993).

Por otro lado, es importante recordar que todas las personas tienen derecho a sentirse apoyadas para desarrollar canales de comunicación adecuados, lo cual facilitará el uso de esta estrategia con un mayor grado de funcionalidad, además, en una interacción donde uno de los interlocutores ha desarrollado una mejor referencia a la comunicación, debe apoyar a los otros a poner significado a su accionar.

Lo anterior lo lleva a cabo el interlocutor al combinar los canales de comunicación con la imaginación, sin embargo, no siempre debe improvisar, sino más bien debe escoger y 
organizar cuidadosamente todo proceso de interacción y de dar sentido al intento comunicativo de la persona con autismo.

La situación antes descrita, resulta compleja para las personas con autismo si se parte de la idea que desarrollaron Baron-Cohen, Leslie y Frith (1985) sobre la Teoría de la Mente, quienes comentaban que este concepto hace referencia a la capacidad de atribuir estados mentales a otros. Como se puede ver esta capacidad es importante en la expresión y la comprensión de la interacciones sociales, y es común que los niños ya a partir de los dos años la desarrollen, y es hacia los cuatro años cuando los niños sin discapacidad la presentan; en este sentido, las personas con Autismo no habrían desarrollado esa capacidad, posiblemente, por el trastorno generalizado del desarrollo con el cual cursan durante toda su vida.

Por estas razones es importante trabajar comunicación y lenguaje en personas con autismo, pero siempre y cuando se tenga en cuenta reforzar todos los intentos comunicativos que ellas tienen en su entorno.

\section{3.- La comunicación en personas con autismo}

Para comprender la comunicación en personas con autismo es necesario conocer el proceso de comunicación en el desarrollo normal, muchas veces se considera que las personas con autismo porque hablan pueden tener un nivel de competencia comunicativa similar o igual al del habla, sin embargo, esto no es cierto, aun cuando estas personas no cursen con un compromiso en el nivel cognitivo.

Por lo tanto, según anotan autores como Frith (1989); Gortázar y Tamarit (1989); Gortázar (1990),, Gortazar (1993), Roth (1990), Soto (1994), Schaeffer (1986), entre otros, la alteración en la comunicación de las personas con autismo sobrepasa la posibilidad que ellas tienen de poder hacer uso del lenguaje hablado, y esa dificultad se centra de alguna manera en un aspecto de tipo pragmático, o sea en el conjunto de reglas que rige el uso del lenguaje en el conjunto de interacciones sociales que llevan a cabo las personas, lo cual requiere que las personas que interactúan puedan atribuir estados mentales unas a otras y que esto, les permita un proceso de retroalimentación, de esta manera se esperaría que una persona con autismo al interactuar con otra persona pueda tener en cuenta su estado, sus 
intenciones, sus deseos, sus creencias, entre otros aspectos para así poder llevar a cabo intercambios comunicativos fluidos, situación que se ve truncada por los problemas de metarepresentacionalidad de las personas con Autismo (Sotillo y Rivière, 1997).

Lo anterior puede determinarse al observar un acto comunicativo de una persona con Autismo, a saber, ellas a menudo no dan muestras de intercambio fluido, utilizan un tono monocorde, sus gestos faciales no están acordes con lo que están expresando, aspectos que las personas sin la condición autista (u otra condición discapacitante) más bien se empeñan en disminuir o resaltar para favorecer sus intercambios comunicativos. Estas situaciones pueden verse reforzadas en el caso de personas que además del autismo presentan algunos compromisos a nivel cognitivo (Sotillo y Rivière, 1997, Soto, 1994).

En este sentido, hay estudios que han demostrado que muchas de las personas con autismo acostumbran a tener "intentos comunicativos" mas de tipo de demanda, que de "declaración".

De alguna manera, según comenta Soto (1994) la presencia de lo que Bates denomina "protoimperativos" más que de "protodeclarativos" tiene mucha relación con los esquemas instrumentales triangulares interactivos que se establecen entre el niño, un adulto y un objeto. Por lo tanto, la dificultad que se da en la comunicación de las personas con autismo, está especialmente definida por la mayor presencia de intentos de comunicación de demanda, que de intentos de comunicación declarativa, ya que son estos últimos sobre los que se basan las diferentes interacciones de las personas, y se llevan a cabo los procesos comunicativos, como intercambio.

\section{4.- Hacia donde deben dirigirse los esfuerzos en la comunicación y el lenguaje}

Los esfuerzos de las personas que realizan labores educativas en personas con autismo, deben dirigirse más hacia los aspectos de comunicativos que hacia la adquisición del lenguaje, sin que esto se convierta en tarea única y exclusiva del terapista del lenguaje, ya que como se ha venido desarrollando desde el inicio la comunicación debe concebirse como una tarea social, y debe ser un hilo conductor de todo el trabajo que se lleva a cabo por los diferentes profesionales. 
En el caso de las personas con Autismo es importante que el trabajo en comunicación se convierta en un proceso estructurado libre y espontáneo, la persona debe aprender a entender y usar los códigos, la idea es despertar en ella la iniciativa de comunicación espontáneo, esto le facilitará la satisfacción de necesidades y facilitará los procesos de interacción con las personas del entorno (Sotillo y Rivière, 1997, Soto, 1994, Gortazar, 1993, Tamarit, 1988 a).

Para involucrarse en el trabajo en el área de la comunicación de personas con autismo, será necesario llevar a cabo algunos pasos, a saber;

a. Llevar a cabo observaciones y evaluaciones de la forma en que se comunica la persona con autismo con su docente,

b. Realizar observaciones y evaluaciones de las formas en que reacciona la persona con autismo a los mensajes de sus compañeros,

c. Observar y evaluar los momentos en que el niño es quien toma la iniciativa de comunicarse (Sotillo y Rivière, 1997, Soto, 1994).

Lo anterior permitirá determinar en que momentos o situaciones la persona con autismo se comunica mejor, y ante cuales situaciones es capaz de establecer comunicación con los demás.

De esta manera, se puede en un primer momento apoyar estos momentos y facilitar situaciones de interacción y a partir de aquí, se podría entonces implementar y enseñar a las personas con autismo algún código de comunicación.

Se persigue así, la creación de la conducta comunicativa expresiva espontánea, y no necesariamente a que se comprenda o que responda ante las acciones de los demás.

La idea es partir de la comunicación pero como comunicación social, de tal manera que se desarrolle una secuencia de acción y reacción, ya sea a la presencia de actos o gestos o al uso del lenguaje. Trabajando de esta forma los profesionales que trabajen con la persona con autismo darán intención y significado a sus acciones ya sean que estén en su repertorio o aquellas que se le están enseñando, y esto se logra cuando las personas con autismo observan la reacción en los demás o en el entorno, logrando de esta manera establece una 
relación causal entre su acto y la respuesta que da el entorno, se desarrolla de esta manera la causalidad social (Sotillo y Rivière, 1997, Soto, 1994).

Cuando se da el caso de personas con autismo que además tienen algún compromiso a nivel cognitivo, y que como comentaba Soto (1994) al citar a Carr que aproximadamente el cincuenta por ciento de las personas con autismo son no verbales, se hace necesario el uso de los Sistemas Alternativos de Comunicación, y aquí merece especial atención el programa de Comunicación Total de Schaeffer et al. (citado y discutido ampliamente en Soto, 1994).

La idea del método no es solamente enseñar un signo o una seña, lo que se pretende es favorecer una estrategia de relación interpersonal, una estrategia de interacción social en la cual se le enseña a la persona con autismo a dirigirse al adulto para así conseguir lo que desea, o sea que se le enseña a la persona con autismo que una acción suya diferenciada que le ha sido enseñada obtiene una reacción predecible del entorno, y además esta reacción es provocada por su acción, en este caso el signo, de esta manera, logra esta persona obtener una herramienta con la cual puede regular intencionalmente el entorno por medio del adulto (Soto, 1994).

De lo anterior se resume que muchas veces más allá de enseñar los signos es necesario enseñar esquemas de percepción de causalidad tanto en lo referente a los aspectos físicos como a los sociales, además de estrategias de anticipación y estrategias instrumentales.

\section{5.- $\quad$ El método Schaeffer et al. (1980)}

Según anotan Sotillo y Rivière (1997); Soto, (1994), entre otros autores, la fuerza del Programa reside no tanto en el hecho de que el soporte alternativo a la palabra sean los signos, sino principalmente en el procedimiento específico de enseñanza de ese soporte.

Un procedimiento de aprendizaje sin error, encaminado directamente a la enseñanza, por un lado de pautas de comunicación expresiva (los signos) y por otro, pero indisoluble del anterior, de funciones comunicativas. Esta enseñanza, inseparable, de topografías (signos) y de funciones, por medio del procedimiento de análisis conductual denominado moldeamiento y la técnica del encadenamiento marcha atrás, la cual permite el la adquisición de cada signo 
que se enseña y su uso espontáneo generalizado, lo cual redunda en un uso funcional de los signos, ya que la persona mediante su uso logra ver satisfechas sus necesidades.

También el uso del procedimiento denominado aprendizaje sin error, en el cual no se atienden los errores de las personas, para que ellas no vean reforzados sus errores, sino que más bien, las personas ven reforzadas sus intenciones comunicativas acertadas, mediante la consecución de aquello a lo que hace referencia su signo o intento comunicativo.

Por otro lado se adaptan los objetivos al nivel evolutivo de las personas con autismo (Gortazar, 1993, Peeters, 1997).

Por otro lado, según Sotillo y Rivière (1997) y Soto (1994), el método también requiere que los estudiantes con autismo hayan aprendido los requisitos de las conductas que se van a enseñar.

Asegurar la adquisición previa a los requisitos de conducta que se pretenden enseñar. Los docentes deben tener claro que es necesario realizar análisis de tareas que le permitan al estudiante el logro de los objetivos propuestos. En caso de que sea necesario se debe brindar la ayuda necesaria ya sea esta como instigación física o verbal.

También es importante dirigir la acción en los primeros momentos para evitar el error, además de que es necesario llevar a cabo un buen control de estímulos, de tal manera que se evite la distracción, la ambigüedad y la agotabilidad de la persona con la que se trabaja.

Al la persona se le debe mantener motivada mediante el desarrollo de actividades llamativas y participativas, lo mismo que el uso de reforzadores muy potentes. Todo trabajo que se desarrolle debe resultar importante, y atractivo para las personas con las que se trabaja.

Como mencionan Sotillo y Rivière (1997) y Soto (1994), es importante recordar que el aprendizaje con este método se produce de manera incidental, o sea que los episodios de enseñanza son iniciados por las personas con autismo, de tal manera que la tarea de las personas que trabajan con él es determinar el movimiento natural que utiliza el niño con alguna intención comunicativa, para posteriormente utilizar el moldeamiento de la forma y la 
ubicación correcta de la mano, y junto con el niño mediante la instigación realizar tantos movimientos como sílabas tenga la palabra que se trabaja. Toda vez que la persona haga los movimientos necesarios se le refuerza inicialmente con el objeto o situación que solicita o emite, para que de esta manera el niño pueda establecer la relación de causalidad.

\section{6.- Otras formas de abordaje de la comunicación y el lenguaje}

6.1.- Es importante recordar que cualquier proceso de trabajo que se realice en la comunicación y el lenguaje debe responder a objetivos muy claros del desarrollo, a saber

1. La atención

2. La imitación

3. La activación de la motricidad orofacial

4. La recepción de información de tipo multisensorial

5. La comprensión y

6. La expresión o producción.

Cuando se quiere trabajar con las personas no verbales con autismo se debe abordar en un primer momento los prerrequisitos o campos de habilidades para el aprendizaje, ver, escuchar y moverse, esto se logra mediante ejercicios de atención, imitación y seguimiento de órdenes.

Luego se pasaría a hacer conciencia de los órganos fonoarticulatorios, para posteriormente pasar a la articulación de los fonemas y los sinfones; y la enseñanza de elementos prosódicos del habla, a saber, la entonación, las pausas, el ritmo y el tono, posteriormente se desarrolla la estructuración progresiva de la frase.

En otro momento del trabajo se lleva a cabo el desarrollo de los componentes semánticos, esto se lleva a cabo principalmente cuando se aumenta el vocabulario de la persona.

El último componente el cual es muy importante se denomina pragmático consiste en enseñarle a la persona el uso social del lenguaje. 
Cuando la persona con la que se trabaja ya tiene lenguaje, el procedimiento es partir del nivel que tiene y tomar en cuenta los aspectos anteriores.

6.2.- Otra estrategia de intervención es la denominada Paradigma del lenguaje natural (PLN) (Koegels et al., en Gortázar, 1990) el cual implica

1.- uso de estímulos funcionales escogidos por el niño,

2.- reforzadores naturales,

3.- reforzar los intentos de comunicación aun cuando estos no sean correctos (aproximaciones),

4.- ensayos de la estrategia dentro de los intercambios comunicativos.

6.3.- Existen otros métodos basados principalmente en el uso de pictogramas, imágenes y otros elementos comunicativos, principalmente tomando en cuenta sistemas alternativos y aumentativos de comunicación.

\section{7.- A manera de cierre}

Al ser el lenguaje y la comunicación una de las características centrales que conlleva el Síndrome Autista es necesario buscar una respuesta a las necesidades de cada una de las personas y sus familias, favoreciendo de esta manera que las intenciones comunicativas de ellas, se vea enriquecida, sin embargo no hay que perder de vista el carácter interactivo de la comunicación, y por lo tanto, eje principal de cualquier trabajo que se lleve a cabo con las personas portadoras del Síndrome.

Por otro lado es necesario el trabajo interdisciplinario de tal manera que la persona con Autismo sea abordada de manera integral, y que se satisfagan sus necesidades, intereses y características, a partir de sus potencialidades; el trabajo por lo tanto no es solo del docente, o del terapista del lenguaje, es también del padre de familia y de la familia en general, por lo que es necesario brindar múltiples experiencias de intentos comunicativos, en los cuales las personas tengan la posibilidad de hacer uso de todo aquello que aprenden en los encuentros comunicativos, ya sea con las personas cercanas a su entorno, en el espacio áulico o en su comunidad. 
Todos los que nos involucramos en esta aventura debemos propiciar la comunicación de las personas con autismo, para que ellas le encuentren sentido al lenguaje y a la comunicación, como experiencia a partir de la cual ve satisfecha sus necesidades, intereses e intenciones. El trabajo es arduo, pero es importante llevarlo a cabo, si queremos que las personas con Autismo participen en igualdad y equiparación de oportunidades.

\section{REFERENCIAS}

Atam-Fundesco. (1988). Manual de toma de decisiones y de evaluación para el aprendizaje y uso de los sistemas aumentativos de Comunicación. Unidad de Comunicación Aumentativa. Madrid: Atam-Fundesco..

Baumgart, D., Jchnsan, J. y Hekmstetter, E. (1996) Sistemas Alternativos de Comunicación para personas con discapacidad. Madrid: Ed. Cast., Alianza Editorial, S.A.

"Diccionario de Signos para alumnos con grave retraso en el desarrollo". Varios autores. C.P. de E.E. "Pérez Urruti". Churra. Murcia.

Dubois, G. (1996) "Lenguaje y Comunicación". Madrid: Ed. Masson.

Frith, U. (1989) Autism: Explaining the Enigma. Oxford: Blackwell.

García Cernuda, J. P.; Gortázar, M. y Bernardo M. (1986). Procedimientos de Comunicación simultánea. Consideraciones sobre la evaluación previa. En Monfort: Investigación y Logopedia. III Simposio de Logopedia. Madrid: CEPE.

Gortázar y Tamarit. (1989). Lenguaje y comunicación. Madrid: Centro Nacional de Recursos para la Educación Especial.

Gortázar, xxxx. (1990). Ecolalia y adquisición del lenguaje en niños autistas: implicaciones de cara a la intervención. VI Congreso Nacional de Autismo (AETAPI) Palma de Mallorca.

Gortázar (1993) Implicaciones del modelo de enseñanza natural del lenguaje en la intervención de personas con autismo. En Canal, B. R., Crespo, C. M., Pérez, P. Y., Sanz, V. T., y Verdugo, A. M. A. (1993) El autismo 50 años después de Kanner (1943). Actas del VII congreso Nacional de Autismo. Salamanca, España: Amarú Ediciones.

Marchena, C. (1998) "El trastomo autista, contextualización e intervención logopédica". Madrid: Ed. Alfar.

Martos, J. (1997), Explicaciones psicológicas y Tratamiento educativo en autismo. En A. Riviére y J. Martos (comp.), El tratamiento del Autismo. Nuevas Perspectivas. Madrid: IMSERSO. 
Riviére, A. (1997), El tratamiento del autismo como Trastorno del desarrollo: Principios Generales. En Riviére y Martos, El tratamiento del Autismo. Nuevas Perspectivas. Madrid: IMSERSO.

Riviére A. (2001) Lenguaje y Autismo. En Autismo: Enfoques actuales para padres y profesionales de la salud y la educación, Tomo 2. Madrid: Fundec. Serie Autismo.

Roth (1990) Autism. En Introduction to Psychology. Vol 2. London: Lawrence Erlbaum

Schaeffer, B (1982b). "Linguistic functions and language intervention: part II. Special topic". Journal of special education, 16, 401-411.

Schaeffer, B (1982a). "Linguistic functions and language intervention: part I. Concepts, evidence, and instructional sequence". Journal of special education, 16, 289-308.

Programa de Comunicación Total. Traducción al español del libro "Total Comunication A Signed Speech Program for Nonverbal Children", Benson Schaeffer, Arlene Musil, George Kollinzas (1980). Champaing, Illinois: Research Press.

Schaeffer, B (1980) "Teaching Signed Speech to non-verbal Children: Thecsy and method". Sign language studies 26, 29-63.

Lenguaje verbal espontáneo para niños autistas mediante el Habla Signada. (Traducción). Schaeffer, B; Musil, A; Kollinzas, G. y McDowell, P. (1977): "Spontaneous verbal language for autistic children through signed speech". Sign language Studies, 17, 287328.

Schaeffer, B. (1986): Lenguaje de signos y Lenguaje oral para niños minusválidos. "Lenguaje de signos y lenguaje oral para niños minusválidos". En Marc Monfort (Ed.): Investigación y logopedia. Madrid: CEPE.

Comunicación Total; Programa de B. Schaeffer. Centro Nacional de Recursos para la Educación Especial. "Intervención Educativa en Autismo Infantil. Serie Formación.

"Programa de Comunicación Total: Su influencia en el desarrollo general del niño". Tamarit, J. (1986). Actas del IV Congreso de Terapeutas de Autismo (AETAPI). Ayuntamiento de Valladolid. Valladolid.

Sotillo, M. (Ed.) (1993): "Los sistemas alternativos de comunicación". Madrid: Trotta.

Sotillo, M. (2001) Comunicación y Lenguaje en Autismo. Sistemas Alternativos de Comunicación. Madrid: Fundec. Serie Autismo. Tomo II.

Sotillo, M. y Rivière, A. (1997) Sistemas Alternativos de comunicación y su empleo en autismo. En: Rivière, A. y Martos, J. (comp) (1997). El tratamiento del Autismo: Nuevas perspectivas. Madrid: Ministerio de Trabajo y Asuntos Sociales, Secretaría General de Asuntos Sociales. Instituto de Migraciones y Servicios Sociales.

Soto, R. (1994) Un sistema alternativo en la enseñanza de lenguaje a niños con autismo: Comunicación Total. Universidad de Costa Rica, Facultad de Educación, 
Escuela de Formación Docente. Tesis para optar al grado de Licenciado en Educación Primaria.

Soto, C. R. (2002) Didáctica del Lenguaje y de los Estudios Sociales para Personas con Necesidades Educativas Especiales. San José, Costa Rica: Editorial Universidad Estatal a Distancia.

Peeters, T. (1997) El autismo y los problemas de comprender y utilizar símbolos. En: Rivière, A. y Martos, J. (comp) (1997). El tratamiento del Autismo: Nuevas perspectivas. Madrid: Ministerio de Trabajo y Asuntos Sociales, Secretaría General de Asuntos Sociales. Instituto de Migraciones y Servicios Sociales.

Tamarit., J. (1988 a) "Sistemas alternativos de Comunicación en Autismo: Algo más que una alternativa". Alternativas para la comunicación, 6,3-5.

Tamarit, J. (1988). Centro de Recursos. "Comunicación Simultánea; intervención en deficiencia mental", Seminario internacional sobre lenguaje de sigynos en la educación de los niños con dificultades de comunicación oral. Madrid, Mayo-Junio.

Tamarit, J. (1988). "Los trastornos de la comunicación en deficiencia mental y otras alteraciones evolutivas: Intervención mediante sistemas de comunicación total". En Carmen Basil y Ramón Puig (Eds.): Comunicación Aumentativa INSERSO, Col. Rehabilitación. Madrid.

Tamarit, J. (1993):"La aportación del Programa de Comunicación Total de Schaeffer y sus cols. a la EE de nuestro país". Actas del VII Congreso Nacional de Autismo. Amarú Ediciones -Salamanca.La mejora de la enseñanza del Lenguaje para niños autistas de B. Schaeffer". Actas del VII Congreso Nacional de Autismo. Amarú Ediciones. Salamanca

Tamarit, J. (1993). Sistemas alternativos de Comunicación en los trastornos generalizados del desarrollo. Documentos CEPS. Centro de Profesores de Murcia. "Glosario de signos para alumnos con autismo y con otras alteraciones". Varios autores. Edt. Centro de Profesores. Madrid-Centro.

Tamarit, J. y Gortázar, P. (1988): "Modelo explicativo de las alteraciones comunicativas en autismo desde la perspectiva de la cognición social". V Congreso Nacional de Autismo. (AETAPI). Cádiz.

Von Tetzchner S. y Martinsen, H. (1993): "Introducción a la enseñanza de signos y al uso de ayudas técnicas para la comunicación". Visor. Madrid. 